\title{
Evidência e análise de vulnerabilidades de pessoas com Diabetes Mellitus: potencialidades para o cuidado
}

\author{
Evidence and analysis of vulnerability of people with Diabetes Mellitus: potential for care \\ Evidencia y análisis de vulnerabilidades de personas portadoras de Diabetes Mellitus: \\ potencialidades de atención
}

\author{
Anna Carolina dos Santos Chaves ${ }^{I}$; Lina Márcia Miguéis BerardinelliII; Nathália Aparecida da Costa Guedes ${ }^{\text {III; }}$ \\ Clícia Vieira Cunha ${ }^{I V}$; Mauro Leonardo Salvador Caldeira dos Santos ${ }^{V}$
}

\begin{abstract}
RESUMO: Objetivou-se evidenciar e analisar vulnerabilidades de pessoas com Diabetes Mellitus tipo 2 descritas na produção científica da enfermagem. Revisão integrativa orientada pela questão: Como se expressa a vulnerabilidade de pessoas com Diabetes Mellitus tipo 2 na literatura da enfermagem? Pesquisados artigos publicados entre 2005 a 2015, nas bases de dados Biblioteca Virtual de Saúde, Literatura Latino-Americana e do Caribe em Ciências da Saúde, Medical Literature Analysis and Retrieval System Online, Cumulative Index to Nursing and Allied Health Literature, a partir da conjugação das palavras-chave vulnerabilidade, enfermagem e Diabetes Mellitus tipo 2, totalizando oito artigos selecionados. Os artigos foram agrupados por área temática, emergindo duas categorias: Vulnerabilidade e a condição humana; Enfrentamento do diabetes com educação em saúde. Acredita-se que a compreensão das vulnerabilidades contribuirá para redimensionamento do cuidado em saúde de pessoas com Diabetes Mellitus, de forma a incorporar os pressupostos do cuidado integral, incluindo as dimensões do bem-estar biopsicossocial.
\end{abstract}

Palavras-Chave: Diabetes Mellitus tipo 2; vulnerabilidade em saúde; enfermagem; promoção da saúde.

\begin{abstract}
This study to demonstrate and examine the vulnerabilities of people with type 2 Diabetes Mellitus as described in scientific nursing production used integrative review guided by the question: How is the vulnerability of people with type 2 diabetes mellitus expressed in the nursing literature? The Virtual Health Library, Latin American and Caribbean Health Sciences Literature, Medical Literature Analysis and Retrieval System Online, and the Cumulative Index to Nursing and Allied Health Literature were searched, using the combination of keywords 'vulnerability', 'nursing', and 'Diabetes Mellitus type 2' for articles published from 2005 to 2015. When the 8 articles selected were grouped by subject area, two categories emerged: vulnerability and the human condition; and coping with diabetes with health education. It is believed that an understanding of the vulnerabilities will contribute to rescaling health care for people with diabetes mellitus, in such a way as to incorporate the tenets of comprehensive care, including the dimensions of bio-psycho-social welfare. Keywords: Type 2 Diabetes Mellitus; health vulnerability; nursing; health promotion.
\end{abstract}

RESUMEN: El objetivo fue poner en evidencia y analizar las vulnerabilidades de las personas portadoras de Diabetes Mellitus tipo 2 descritas en la producción científica de enfermería. Revisión integradora orientada por la pregunta: ¿Cómo se expresa la vulnerabilidad de personas portadoras de Diabetes Mellitus tipo 2 en la literatura de enfermería? Fueron investigados artículos publicados entre 2005 y 2015, en las bases de datos de la Biblioteca Virtual de Salud, Literatura Latinoamericana y del Caribe en Ciencias de la Salud, Medical Literature Analysis and Retrieval System Online, Cumulative Index to Nursing and Allied Health Literature, a partir de la combinación de las palabras clave: vulnerabilidad, enfermería y Diabetes Mellitus tipo 2. En total, se han seleccionado totalizando 8 artículos. Los artículos fueron agrupados por áreas temáticas, emergiendo dos categorías: la vulnerabilidad y la condición humana; enfrentamiento de la diabetes con educación en salud. Se cree que la comprensión de las vulnerabilidades contribuirá a redimensionar el cuidado en salud de personas con Diabetes Mellitus, de manera a incorporar los presupuestos de la atención integral, incluyendo las dimensiones del bienestar biopsicosocial. Palabras Claves: Diabetes Mellitus tipo 2; vulnerabilidad en salud; enfermería; promoción de la salud.

\section{INTRODUÇÃO}

Nos diferentes processos de transição dos ciclos da vida, os seres humanos estão expostos a vulnerabilidades, sendo que cada uma delas se expressa de maneira diferente, em função da faixa etária em que se encontram, dos contextos nos quais vivem, podendo acarretar problemas de toda a ordem. As implicações podem repercutir na saúde, bem-estar e na qualidade de vida, ocasionando transtornos individual, social,

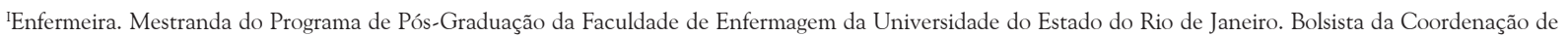
Aperfeiçoamento em Pessoal de Nível Superior. Rio de Janeiro, Brasil. E-mail: carolchavesri@gmail.com.

IEnfermeira. Professor Adjunto do Departamento de Enfermagem Médico-Cirúrgica e do Programa de Pós-Graduação da Faculdade de Enfermagem da Universidade do Estado do Rio de Janeiro. Brasil. E-mail:1.m.b@uol.com.br.

IIIEnfermeira. Mestranda do Programa de Pós-Graduação da Faculdade de Enfermagem da Universidade do Estado do Rio de Janeiro. Bolsista da Coordenação de Aperfeiçoamento em Pessoal de Nível Superior. Rio de Janeiro, Brasil. E-mail: nathyyguedes@gmail.com.

IVEnfermeira. Mestranda do Programa de Pós-Graduação da Faculdade de Enfermagem da Universidade do Estado do Rio de Janeiro. Brasil. E-mail: cliciarebello@gmail.com. vEnfermeiro. Professor Associado III Escola de Enfermagem Aurora Afonso Costa, Universidade Federal Fluminense. Niterói. Brasil. E-mail: mcaleo@uol.com.br. 
gastos financeiros, gerando momentos de avanços e retrocessos, nos quais os indivíduos tornam-se vulneráveis às diversas condições, que podem dificultar $\mathrm{o}$ controle e melhora da doença ${ }^{1,2}$.

Dessa maneira, a vulnerabilidade às doenças difunde-se de modo diferente, dependendo da região, dos grupos sociais e indivíduos a serem avaliados, de modo que está relacionado com o grupo social, o nível de escolaridade, o acesso aos serviços de saúde, entre outros².

$O$ conceito polissêmico de vulnerabilidade expressa, sobremaneira, a transversalidade desse tema em diferentes campos do saber. Em especial, na área da saúde, que vem articulando o cuidado fundamentado em teorias humanísticas, à luz do desenvolvimento humano, no enfrentamento de suas situações de saúde, principalmente no que diz respeito à saúde de pessoas com Doenças Crônicas Não Transmissíveis (DCNT) ${ }^{1}$.

Nesse sentido, a vulnerabilidade, além de consistir em um conjunto de fatores de natureza biológica, epidemiológica, social e cultural, poderá ampliar ou reduzir o risco ou a proteção à determinada patologia, condição ou dano. Está atrelada a fatores individuais e coletivos, intrínsecos e extrínsecos, que podem gerar maior susceptibilidade aos agravos de saúde ou maior capacidade de enfrentamento do indivíduo à doença $\mathrm{a}^{2,3}$; portanto, possui três componentes interligados: individual, social, programático e institucional ${ }^{2}$.

O componente individual inclui a qualidade das informações que o indivíduo possui sobre o problema, a capacidade de elaborar e incorporar informações em seu cotidiano, assim como o interesse e as possibilidades de transformá-las em práticas que visem à proteção e prevenção. $\bigcirc$ componente social refere-se à obtenção dessas informações, que dependem não só dos indivíduos, mas também do acesso aos meios de comunicação, educação, disponibilidade de recursos e à superação de barreiras culturais. $\mathrm{O}$ componente programático inclui os recursos que os indivíduos necessitam para não se exporem a situações de maior risco, das políticas públicas de prevenção e controle das enfermidades, disponibilização de insumos necessários à proteção, compromisso das instituições e dos programas nos diferentes níveis de atenção ${ }^{2}$.

A vulnerabilidade diferencia-se do risco, por seu caráter não probabilístico, uma vez que o risco tenta expressar as chances matemáticas de adoecimento de um indivíduo, quando portador de características específicas, e a vulnerabilidade expressa o potencial de adoecimento/não relacionado a todo e qualquer indivíduo que viva sob determinado conjunto de condições ${ }^{3,4}$.

No âmbito da saúde, o Diabetes Mellitus (DM) é um grande problema crônico de maior repercussão nas discussões de saúde mundial. Dados substancialmente comprovados têm demonstrado que as pessoas se encontram vulneráveis e necessitam de acompanhamento; no entanto, na enfermagem, nem sempre os resultados divulgados na produção científica da área têm apontado as evidências e ações necessárias, a fim de minimizar os problemas de saúde dessas pessoas ${ }^{3}$.

DM é um transtorno metabólico de etiologias heterogêneas, caracterizado por hiperglicemia e distúrbios no metabolismo de carboidratos, proteínas e gorduras, resultantes de defeitos da secreção e/ou da ação da insulina ${ }^{4,5}$.

A última estimativa, divulgada em abril de 2013, indica que, no Brasil, existem mais de 12 milhões de pessoas afetadas pela doença. No ano de 2010, o DM foi responsável pela morte de 54 mil brasileiros, número este quatro vezes maior que os óbitos causados pela Síndrome da Imunodeficiência Adquirida (SIDA) (12 mil) e pelos acidentes de trânsito (42 mil), por exemplo ${ }^{6}$.

Em 2014, a Organização Mundial de Saúde (OMS) registrou que a prevalência da doença estava em torno de $8,3 \%$ da população mundial e que, em 2030, ela será a sétima causa de morte ${ }^{5,6}$. A International Diabetes Federation (IDF) projeta que, até o ano de 2035, os casos mundiais da doença atinjam um patamar alarmante de 592 milhões $^{6}$. Nesse sentido, considera-se um problema de saúde pública de prevalência global, em franca expansão em praticamente todas as regiões do planeta, configurando-se, progressivamente, como uma pandemia, a partir das últimas décadas do século vinte ${ }^{5,6}$.

Embora o Diabetes Mellitus tipo 2 (DM2) seja uma doença que afeta, principalmente, adultos mais velhos, constata-se que, com o aumento crescente da obesidade, a idade de início da doença tem afetado grupos etários mais jovens, o que sugere que o diabetes tornar-se-á uma das doenças mais comuns na população em idade produtiva ${ }^{5,7}$. Esse fato gera momentos de avanços e retrocessos, nos quais os indivíduos tornam-se vulneráveis às diversas condições, que podem dificultar o controle, o acompanhamento e melhora da doença.

Assim, esse trabalho visa indicar as evidências da vulnerabilidade de pessoas com DM2 descritas na produção científica da enfermagem, de forma a contribuir para a atualização de enfermeiros, ampliar as discussões teóricas do cuidado, potencializar reflexões para prática do cuidado em saúde, bem como colaborar na construção de novos objetos de pesquisas.

Para tanto, os objetivos do estudo foram: evidenciar e analisar a vulnerabilidade de pessoas com DM2 na produção científica da enfermagem.

\section{Metodologia}

Trata-se de uma revisão integrativa, cujo método estuda abordagens variadas, reunindo literatura teórica e empírica, com o objetivo de ampliar a compreensão e entendimento sobre um fenômeno particular ou problema de saúde ${ }^{8}$. A categorização dos estudos e a disposição dos achados podem ser realizadas por meio 
de construção de um instrumento que possibilite a sistematização da pesquisa, demonstrando uma quantidade expressiva de dados que serão analisados e facilitarão a avaliação individualizada de cada trabalho ${ }^{8,9}$.

Além disso, permite gerar conhecimento atualizado sobre um determinado problema de pesquisa e verificar se o conhecimento é válido para ser transferido para a prática; portanto a construção da revisão integrativa deve seguir padrões de rigor metodológico, que possibilitarão ao leitor identificar as características dos estudos analisados e oferecer embasamento para o avanço em enfermagem ${ }^{10}$.

Assim, foram seguidos os seguintes passos: delimitação da questão para a revisão; estabelecimento dos critérios amostrais; definição das características da pesquisa; análise dos dados; interpretação dos resultados e, por último, apresentação da revisão ${ }^{9,10}$.

A questão selecionada foi: Como se apresenta a literatura em enfermagem acerca das vulnerabilidades de pessoas com DM2? Em seguida, foram estabelecidos os critérios de seleção do estudo: artigo completo, periódico qualificado, resultados de pesquisas, relatos de experiência, revisão e reflexão nos idiomas português, inglês ou espanhol, disponíveis no meio eletrônico, gratuitamente, no recorte temporal dos últimos 10 anos (2005-2015). Foram excluídos do estudo textos que não se enquadravam no tema proposto e no recorte temporal, artigos repetidos, resumos de anais, dissertações, teses, monografias, livros, relatórios, entre outros documentos.

A coleta de dados foi realizada no mês de abril de 2015, nas seguintes bases: Biblioteca Virtual de Saúde (BVS), no modo integrado com a Base de Dados de Enfermagem (BDENF), Literatura Latino-Americana e do Caribe em Ciências da Saúde (LILACS), Medical Literature Analysis and Retrieval System Online (MEDLINE) e na Cumulative Index to Nursing and Allied Health Literature (CINAHL), por intermédio dos Periódicos Capes. Foram utilizadas as seguintes palavras-chave combinadas: vulnerabilidade, enfermagem e DM2.

O estudo de revisão integrativa, como instrumento da prática baseada em evidências, caracteriza-se por ser uma abordagem que se direciona tanto para o cuidado clínico quanto para o ensino fundamentado no conhecimento e na qualidade da evidência científica da prática clínica ${ }^{10}$.

Para tanto, as evidências são classificadas de forma hierárquica para a avaliação de pesquisas ou outras fontes de informação, baseando-se na categorização da Agency for Healthcare Research and Quality (AHRQ) dos Estados Unidos da América. Sendo assim, a sua classificação apresenta-se em seis níveis, descrita a seguir: nível 1- metanálise de múltiplos estudos controlados; nível 2- estudo individual com desenho experimental; nível 3- estudo com desenho quase experimental como estudo sem randomização com grupo único pré e pós-teste, séries temporais ou caso-controle; nível 4- estudo com desenho não experimental como pesquisa descritiva correlacional e qualitativa ou estudos de caso; nível 5- relatório de casos ou dado obtido de forma sistemática, de qualidade verificável ou dados de avaliação de programas; nível 6- opinião de autoridades respeitáveis baseada na competência clínica ou opinião de comitês de especialistas, incluindo interpretações de informações não baseadas em pesquisas; opiniões reguladoras ou legais ${ }^{10}$.

Após a seleção dos artigos, eles foram dispostos em um instrumento a fim de extrair os dados de cada publicação selecionada, constando as seguintes informações: título, autores, periódico publicado, ano, volume, tipo de pesquisa/metodologia, base de dados utilizada ${ }^{10}$.

Em seguida, os dados foram organizados a partir da técnica de análise temática, que consiste em encontrar os núcleos de sentido que compõem uma comunicação cuja presença ou frequência signifique alguma coisa para o objetivo analítico visado. Nesse sentido, o tema é a unidade de significação que se liberta naturalmente de um texto analisado segundo critérios relativos à teoria que serve de guia à leitura. Para proceder à análise, foi organizado e estruturado o conteúdo dos dados seguindo as fases sequenciais: a pré-análise, a exploração do material e o tratamento dos resultados ${ }^{11}$.

\section{Resultado e Discussão}

Na base de dados BVS, foram encontrados oito $\operatorname{artigos}^{12-19}$, porém somente dois eram pertinentes à temática, no entanto não estavam disponíveis na íntegra. Na base de dados CINAHL, foram encontrados 177 trabalhos, sendo que apenas oito estavam disponíveis na íntegra e contemplavam a temática escolhida. Assim, a amostra final foi constituída por oito estudos, todos da base de dados CINAHL.

Dessa forma, observa-se um número reduzido de artigos científicos disponíveis acerca das vulnerabilidades das pessoas com Diabetes Mellitus tipo 2.

Os oito artigos encontrados foram apresentados segundo o título, os autores, periódico, base de dados e delineamento da pesquisa ${ }^{12-19}$, conforme mostra Figura 1.

No que se refere ao ano de publicação, não foi localizada produção sobre o tema no período de 2005 2009. Verifica-se um número de publicações maior no ano de 2010, com três estudos ${ }^{12,16,18}$, uma em $2011^{19}$, $2012^{17}, 2013^{13}$, seguido por duas em 2015 ${ }^{14,15}$. Quanto à área dos estudos publicados, percebe-se a autoria de um artigo procedente da área de economia ${ }^{12}$, um da medicina ${ }^{15}$, outro da educação física ${ }^{18}$ e cinco artigos da enfermagem ${ }^{13,14,16,17,19}$.

Em relação ao delineamento metodológico da pesquisa, em todas as bases de dados e biblioteca virtual verificam-se três artigos de pesquisas quantitativas $^{12,14,18}$, dois de pesquisa qualitativa ${ }^{13,16}$, um 


\begin{tabular}{|c|c|c|c|}
\hline Título & Autores & $\begin{array}{l}\text { Periódico (ano, } \\
\text { volume) }\end{array}$ & Tipo de pesquisa \\
\hline $\begin{array}{l}\text { The financial vulnerability of } \\
\text { individuals with diabetes }\end{array}$ & $\begin{array}{l}\text { Schofield DJ, Percival R, } \\
\text { Passey ME, Shrestha RN, } \\
\text { Callander EJ, Kelly SJ. }{ }^{2}\end{array}$ & $\begin{array}{l}\text { The British Journal of } \\
\text { Diabetes and Vascular } \\
\text { Disease. v. } 10.2010 .\end{array}$ & Pesquisa quantitativa \\
\hline $\begin{array}{l}\text { Vulnerability in health care - } \\
\text { reflections on encounters in } \\
\text { every day practice }\end{array}$ & $\begin{array}{l}\text { Gjengedal E, Ekra EM, Hol H, } \\
\text { Kjelsvik M, Lykkeslet E, } \\
\text { Michaelsen R, Orøy A, } \\
\text { Skrondal T, Sundal H, Vatne } \\
\text { S, Wogn-Henriksen K. }{ }^{13}\end{array}$ & $\begin{array}{l}\text { Nursing Philosophy. v. } \\
\text { 14. } 2013 .\end{array}$ & $\begin{array}{l}\text { Estudo teórico - } \\
\text { reflexivo }\end{array}$ \\
\hline $\begin{array}{l}\text { Genetic vulnerability to diabetes } \\
\text { and obesity: Does education offset } \\
\text { the risk? }\end{array}$ & $\begin{array}{l}\text { Liu SY, Walter S, Marden J, } \\
\text { Rehkopf DH, Kubzansky } \\
\text { LD, Nguyen T, Glymour } \\
\text { MM. }^{14}\end{array}$ & $\begin{array}{l}\text { Social Science e } \\
\text { Medicine. v. } 127 . \\
2015 .\end{array}$ & Pesquisa quantitativa \\
\hline $\begin{array}{l}\text { Vulnerability to diabetes in Chine- } \\
\text { se: an age- period-cohort analysis }\end{array}$ & $\begin{array}{l}\text { Wong IOL, Cowling BJ, } \\
\text { Schooling CM. }{ }^{15}\end{array}$ & $\begin{array}{l}\text { Annals of Epidemio- } \\
\text { logy. v. } 25.2015 .\end{array}$ & Estudo de coorte \\
\hline $\begin{array}{l}\text { Learning to live with illness: expe- } \\
\text { riences of persons with recent } \\
\text { diagnoses of diabetes mellitus }\end{array}$ & $\begin{array}{l}\text { Kneck A, Klang B, } \\
\text { Fagerberg I. }{ }^{16}\end{array}$ & $\begin{array}{l}\text { Scandinavian Journal } \\
\text { of Caring Sciences. v. } \\
\text { 25. } 2010 .\end{array}$ & Pesquisa qualitativa \\
\hline $\begin{array}{l}\text { Learning to live with diabetes - } \\
\text { integrating an illness or } \\
\text { objectifying a disease }\end{array}$ & $\begin{array}{l}\text { Kneck A, Klang B, } \\
\text { Fagerberg I. }{ }^{17}\end{array}$ & $\begin{array}{l}\text { Journal of Advanced } \\
\text { Nursing. v. 68. } 2012 .\end{array}$ & Pesquisa qualitativa \\
\hline $\begin{array}{l}\text { Physical activity and diabetes: An } \\
\text { application of the theory of } \\
\text { planned behaviour to explain } \\
\text { physical activity for Type } 1 \text { and } \\
\text { Type } 2 \text { diabetes in an adult } \\
\text { population sample }\end{array}$ & $\begin{array}{l}\text { Plotnikoff RM, Lippke S, } \\
\text { Courneya K, Birkett N, } \\
\text { Sigal R. }{ }^{18}\end{array}$ & $\begin{array}{l}\text { Psychology and } \\
\text { Health. v. 25. } 2010 .\end{array}$ & Pesquisa quantitative \\
\hline $\begin{array}{l}\text { Self-care competence in the case } \\
\text { of Brazilian patients with diabetes } \\
\text { mellitus in a multiprofessional } \\
\text { educational programme }\end{array}$ & $\begin{array}{l}\text { Landim CAP, Zanetti ML, } \\
\text { Santos MA, Andrade TAM, } \\
\text { Teixeira CRS. }{ }^{19}\end{array}$ & $\begin{array}{l}\text { Journal of Clinical } \\
\text { Nursing. v. 20. } 2011 .\end{array}$ & $\begin{array}{l}\text { Estudo prospectivo } \\
\text { comparativo }\end{array}$ \\
\hline
\end{tabular}

FIGURA 1: Descrição dos estudos incluídos na revisão integrativa, segundo título, autor (es), periódico (ano, volume), e tipo de pesquisa. 2005-2015.

estudo teórico ${ }^{17}$, um de coorte ${ }^{19}$ e um comparativo ${ }^{15}$. Foram observados ainda os níveis de evidência de cada estudo, conforme especificado na Figura 2.

Após a seleção do material, foi realizada análise temática, mediante leitura flutuante e constituição do corpus dos artigos. A análise teve como objetivo organizar e sumarizar os dados para responder ao problema proposto neste estudo. E a interpretação buscou associar o descrito nos artigos com conhecimentos mais amplos já obtidos sobre o assunto ${ }^{11}$. A partir de então, da proximidade entre os artigos e repetição dos temas entre eles, originaram-se duas categorias: Vulnerabilidade e condição humana e Enfrentamento do diabetes com a educação em saúde. As categorias são analisadas a seguir.

\section{Vulnerabilidade e condição humana}

A vulnerabilidade de pessoas com DM2 foi a temática de maior destaque entre os trabalhos analisados, revelando diversas perspectivas acerca de seu conceito. É um termo comumente usado para descrever os indivíduos ou grupos de indivíduos ${ }^{12-16}$. Consiste no conjunto de fatores de natureza biológica, epidemiológica, social e cultural cuja interação amplia ou reduz o risco ou a proteção contra uma determinada patologia, condição ou dano ${ }^{20-24}$.

Vulnerabilidade é afetada por fatores pessoais, bem como fatores ambientais e sociais. Tendências na sociedade indicam que um número crescente de pessoas vulneráveis cria exigências adicionais sobre

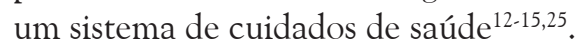

Pessoas ou grupo vulneráveis são definidos como estando em risco de acometimento físico, psicológico e/ou de saúde. Embora todos estejam potencialmente em risco de problemas físicos, e na necessidade de proteção psicológica e/ou de saúde, alguns grupos de pessoas possuem uma maior probabilidade de adoecer. Estes grupos são normalmente identificados como 


\begin{tabular}{|c|c|c|}
\hline Título & Tipo de pesquisa & $\begin{array}{l}\text { Nível de } \\
\text { evidência }\end{array}$ \\
\hline The financial vulnerability of individuals with diabetes ${ }^{12}$ & Pesquisa quantitativa & 4 \\
\hline $\begin{array}{l}\text { Vulnerability in health care - reflections on encounters in every day } \\
\text { practice }^{13}\end{array}$ & $\begin{array}{l}\text { Estudo teórico - } \\
\text { reflexivo }\end{array}$ & 5 \\
\hline Genetic vulnerability to diabetes and obesity: Does education offsetthe risk? ${ }^{14}$ & Pesquisa quantitativa & 4 \\
\hline Vulnerability to diabetes in Chinese: an age- period-cohort analysis ${ }^{15}$ & Estudo de coorte & 4 \\
\hline $\begin{array}{l}\text { Learning to live with illness: experiences of persons with recent diagnoses } \\
\text { of diabetes mellitus }{ }^{16}\end{array}$ & Pesquisa qualitativa & 4 \\
\hline $\begin{array}{l}\text { Learning to live with diabetes - integrating an illness or objectifying a } \\
\text { disease }^{17}\end{array}$ & Pesquisa qualitativa & 4 \\
\hline $\begin{array}{l}\text { Physical activity and diabetes: An application of the theory of planned } \\
\text { behaviour to explain physical activity for Type } 1 \text { and Type } 2 \text { diabetes in an } \\
\text { adult population sample }{ }^{18}\end{array}$ & Pesquisa quantitative & 4 \\
\hline $\begin{array}{l}\text { Self-care competence in the case of Brazilian patients with diabetes } \\
\text { mellitus in a multiprofessional educational programme }{ }^{19}\end{array}$ & $\begin{array}{l}\text { Estudo prospectivo } \\
\text { comparativo }\end{array}$ & 4 \\
\hline
\end{tabular}

FIGURA 2: Descrição dos estudos incluídos na revisão integrativa, segundo o delineamento de pesquisa e os níveis de evidências. 2005-2015.

aqueles que possuem baixo poder aquisitivo, desempregados, moradores de rua, doentes crônicos e pessoas com deficiência, as pessoas com AIDS, adolescentes grávidas, crianças, idosos em situação vulnerável, os imigrantes e os refugiados, e doentes mentais ${ }^{13-15,24-26}$.

Sob a perspectiva fenomenológica, diz respeito ao estado ou condição existencial, considerando fatores intrínsecos e extrínsecos; isto é, torna o ser humano mais vulnerável quando exposto a riscos ou na presença de danos. Frequentemente associada a grupos de pessoas, como crianças e adultos que sofrem de doenças crônicas ${ }^{13,14,16}$.

Como acontece com todas as doenças crônicas não transmissíveis, a DM representa uma interação de fatores genéticos, estilo de vida e fatores ambientais, cuja elucidação facilita a prevenção e o controle da doença ${ }^{12-17}$.

Os artigos sinalizaram que a prevenção da diabetes deve ser uma prioridade, a fim de reduzir os custos pessoais de poupança perdidos e riqueza, além da reconhecida carga de morbidade excessiva e os custos de saúde associados à doença e sua mortalidade ${ }^{12-15,24}$. A vulnerabilidade ao DM2 foi aplicada quanto à genética, estilo de vida e financeira ${ }^{12-15}$.

Evidencia-se que a vulnerabilidade não é mera redução da capacidade de autodeterminação, mas um sentido mais profundo de vulnerabilidade, que é um referencial próprio que se articula, isso sim, com autonomia, com equidade, com prudência, com alteridade ${ }^{15,16,25,26}$.

A vulnerabilidade no indivíduo com diabetes é multifatorial e multifacetada, não apenas restrita a uma questão de redução da capacidade de autodeterminação $\mathrm{O}^{13,14}$. Muda em cada circunstância que o indivíduo se encontre, podendo ter percepções e graus diversos entre os diferentes indivíduos e situações ${ }^{14,17}$.

A compreensão da vulnerabilidade das pessoas com DM2 deve estar embasada na pluralidade dos espaços em que as pessoas vivem, bem como na diversidade de escolha dos indivíduos ${ }^{13,14,16,24-27}$. Esse entendimento subsidiará a mobilização dos profissionais de saúde e população civil na busca de melhores condições de vida e saúde com vistas à transformação social.

\section{Enfrentamento do diabetes com a educação em saúde}

Nota-se, nesta categoria, que os artigos destacam que, apesar dos avanços tecnológicos em saúde, uma minoria de pessoas que convive com o DM2 alcança as metas estabelecidas para o controle da doença. A incapacidade de atingir esses objetivos aumenta o risco de desenvolver complicações médias a graves, bem como experimentar o sofrimento e a angústia de conviver com a diabetes ${ }^{17,18}$.

Sobre isso, há grande variação entre países, relativa à organização da educação do paciente e sua integração no tratamento do diabetes. As pesquisas, durante as últimas décadas, têm indicado que um dos aspectos mais importantes de cuidados com DM na atualidade é o envolvimento ativo das pessoas no seu próprio cuidado ${ }^{17-19,27-29}$.

processo de ensino-aprendizagem inclui um diálogo interno entre o indivíduo e a vida, cujo foco da motivação deve se centrar no apreender uma nova realidade e compreensão do eu, do que pode ser possível alterar nas mudanças de estilo de vida ${ }^{17,18,29,30}$. No entanto, há dissonância entre o nível de conhecimentos adquiridos e uma mudança nos hábitos e as atitudes dos pacientes com DM relacionadas ao autocuidado. Estes achados indicam que os programas educacionais não são consoantes, e, portanto, recomenda-se a melhoria de competências para o diabetes e autogestão ${ }^{18}$. 
A autogestão consiste em um processo contínuo de facilitação do conhecimento, habilidades e capacidades necessárias para o autocuidado na diabetes, sendo um processo de ensino às pessoas para gerirem a sua doença. Acrescentam que este processo incorpora as necessidades, objetivos e experiências de vida da pessoa $^{17-19,28-31}$.

A educação em saúde é outro fator que afeta a vulnerabilidade. Níveis mais elevados de escolaridade estão diretamente associados com melhor saúde, pois as pessoas mais instruídas, geralmente com melhor renda, têm mais acesso aos serviços de saúde e tendem, também, a praticar ações preventivas de doenças e $\operatorname{agravos}^{16-19,29}$

Os profissionais de saúde são importantes contribuintes neste processo de crescimento. Na enfermagem, proteger os pacientes de danos é um elemento essencial e torna possível o desenvolvimento humano ${ }^{16-18,30}$.

Ações de prevenção e promoção da saúde devem ser prioridade, bem como todas as ações programáticas e de acolhimento a toda demanda espontânea ou não do indivíduo ou do grupo. Tais ações devem focar na autonomia, construída a partir da percepção e experiências dos próprios participantes, considerar as histórias de vida das pessoas, seus valores, crenças e também a situação atual, que possibilite monitoramento e reconstrução da situação em saúde dos indivíduos $^{18-20}$. A capacitação constitui-se como delegação de responsabilidade e os profissionais participam como facilitadores deste processo ${ }^{21-24,28-30}$.

$\mathrm{O}$ DM representa um desafio, tanto para a pessoa que possui, quanto para os familiares e equipe de saúde, sendo, portanto, imprescindível que a equipe de saúde adote novas perspectivas para o cuidado individual e coletivo, possíveis por meio de grupos, centrados na autonomia do indivíduo ${ }^{18,22,24}$. Estratégias de proteção à saúde em grupos são comumente utilizadas e fornecem apoio e suporte psicológico para o enfrentamento das vicissitudes do tratamento, promovendo uma maior aceitação do diabetes e, por conseguinte, uma atitude. Essas estratégias são um espaço de reflexão e coconstrução de conhecimento, incentivando a busca ativa de informações e orientações sobre a enfermidade, objetivando o desenvolvimento da autonomia dos indivíduos ${ }^{16-18 ; 27-31}$.

\section{Conclusão}

Esta revisão possibilitou identificar as evidências científicas sobre as vulnerabilidades das pessoas com Diabetes Mellitus tipo 2, contemplando o objetivo inicialmente proposto e o seu desenvolvimento.

A literatura, de um modo geral, tem apontado que as dificuldades apresentadas pelas pessoas diabéticas, para a obtenção de um bom controle metabólico, estão relacionadas à sua adesão a um plano alimentar, ao incremento da atividade física e ao seguimento da terapêutica medicamentosa.

Os achados são sugestivos de que ainda se fazem necessários novos estudos para que se tenha um melhor esclarecimento acerca das dimensões da vulnerabilidade a que estão expostas as pessoas com Diabetes Mellitus tipo 2.

Acredita-se que a melhor compreensão destas vulnerabilidades poderá contribuir para redimensionar o modelo de atenção à saúde com pessoas acometidas pela diabetes, incorporando os pressupostos do cuidado integral que incluem as dimensões do bem-estar biológico, psicológico, social e espiritual - entre outros -, preconizadas no programa de promoção à saúde.

Ressalta-se a necessidade de estudos envolvendo outras metodologias, a fim de ampliar o escopo da vulnerabilidade no DM tipo 2, a exemplo das narrativas de vida como forma de produzir um rastreamento nos discursos dos sujeitos e captar informações qualitativas. Nesse sentido, poder-se-á iluminar o foco das práticas educativas em saúde, estabelecendo vínculos com as pessoas, envolvendo os protagonistas e seus familiares no autocuidado com mais eficiência, além de ampliar a produção científica nacional e internacional sobre a temática abordada.

\section{REFERÊNCIAS}

1.Berardinelli LMM, Santos I, Santos MLSC, Clos AC, Pedrosa GS, Chaves, ACS. Cronicidade e vulnerabilidade em saúde de grupos populacionais: implicações para o cuidado. Rev enferm UERJ; 18:553-8.

2.Ayres J R CM. Vulnerabilidade e avaliação de ações preventivas. São Paulo: Casa da Edição; 1996.

3.Ministério da Saúde (Br). Plano de ações estratégicas para o enfrentamento das doenças crônicas não transmissíveis (DCNT) no Brasil 2011-2022. 2011. [citado em 05 mar 2015]. Disponível em: portal.saude.gov.br/ portal/arquivos/pdf/cartilha plano.pdf.

4.Ministério da Saúde $(\mathrm{Br})$. Secretaria de Atenção à Saúde. Departamento de Atenção Básica. Estratégias para o cuidado da pessoa com doença crônica: diabetes mellitus. Brasília (DF): Ministério da Saúde; 2013.

5.IDH Atlas de Diabetes. $6^{\mathrm{a}}$ ed. Bruxelas, 2014. [citado em 05 mar 2015] Disponível em: https://www.idf.org/ sites/default/files/EN_6E_Atlas_Full_0.pdf.

6.Sociedade Brasileira de Diabetes. São 12 milhões de diabéticos no Brasil. São Paulo: SBD; 2012.

7.Basarglini, RA. As representações sociais e as experiências com diabetes: um enfoque socioantropológico. Rio de Janeiro: Fiocruz; 2011.

8. Souza MT, Silva MD, Carvalho, R. Revisão integrativa: o que é e como fazer [Internet]. Rev Einstein. 2010; [citado em 02 fev. 2015] 8(1):102-6. Disponível em: http://apps.einstein.br/revista/arquivos/PDF /1134-Einsteinv8n1_p102106_port.pdf. 
9.Ganong LH. Integrative reviews of nursing research. Res Nurs Health. 1987 ; 10(1):1- 11. Acess for EBSCO host ${ }^{\circledR}$. 10.Galvão CM, Sawada NO, Mendes IAC. A busca das melhores evidências. Rev esc enferm USP. [Internet]. 2003; 37:43-50. [citado em 02 fev. 2015]. Disponível em: http://www.scielo.br/pdf/reeusp/v37n4/05.pdf.

11.Minayo MCS. O desafio do conhecimento: pesquisa qualitativa em saúde. 14ª ed. São Paulo: Hucitec; 2014. 12.Schofield D, Percival R, Passey M, Shrestha R, Callander E, Kelly S: The financial vulnerability of individuals with diabetes. Br J Diabetes Vasc Dis. 2010, 10:300-4.

13.Gjengedal E, Ekra EM, Hol H, Kjelsvik M, Lykkeslet E, Michaelsen R, et al. Vulnerability in health care: reflections on encounters in every day practice. Nurs Philos. 2013; 14:127-38.

14.Liu SY, Walter S, Marden J, Rehkopf DH, Kubzansky LD, Nguyen T, et al. Genetic vulnerability to diabetes and obesity: does education offset the risk? Soc Sci Med. 2015; (127):150-8.

15.Wong IOL, Cowling BJ, Schooling CM. Vulnerability to diabetes in Chinese: an age-period-cohort analysis. Annals of epidemiology. 2015; 25:34-9.

16.Kneck A, Klang B, Fagerberg I. Learning to live with illness: experiences of persons with recent diagnoses of diabetes mellitus. Scand J Caring Sci. 2011; 25:558-66. 17.Kneck A, Klang B, Fagerberg I. Learning to live with diabetes-integrating an illness or objectifying a disease. J Adv Nurs. 2012 ; 68:2486-95.

18.Plotnikoff RC, Lippke S, Courneya K, Birkett N, Sigal R. Physical activity and diabetes: an application of the theory of planned behaviour to explain physical activity for type 1 and type 2 diabetes in an adult population sample. Psychology \& Health. 2010; (25):7-23.

19.Landim CA, Zanetti ML, Santos MA, Andrade TA, Teixeira CR. Self-care competence in the case of Brazilian patients with diabetes mellitus in a multiprofessional educational programme. J Clin Nurs. 2011; 20:3394-403. 20.Barra DCC, Lanzoni GMM, Maliska ICA, Sebold LF, Schlindwein BH. Processo de viver humano e a enfermagem sob a perspectiva da vulnerabilidade. Acta Paul Enferm. 2010; 23:831-6

21.Oviedo AD, Boemer MR. A pessoa com diabete: do enfoque terapêutico ao existencial. Rev esc en- ferm USP [Internet]. 2009 [citado em 01 Oct 2015]; 43:744-51. Disponível em: http://www.scielo.br/scielo. php? script $=$ sci_arttext $\&$ pid $=$ S0080623420090004 $00002 \& \operatorname{lng}=\mathrm{en}^{-}$. http://dx.doi.org/10.1590/S0080. 62342009000400002.

22.Silva KL, Sena RR, Grillo MJC, Horta NC, Prado PMC. Educação em enfermagem e os desafios para a promoção de saúde. Rev Bras Enferm. 2009; 62:86-91. 23.Espindola BC, Saboia VM, Valente GSC. Programa educativo em saúde e qualidade de vida de indivíduos com diabetes tipo 2: estudo comparativo. UFPE On Line. 2015; 9:351-9.

24.Santos EI. Vulnerabilidade de enfermeiros no cuidado a pacientes com HIV/AIDS: um estudo de representações sociais [dissertação de mestrado]. Rio de Janeiro: Universidade do Estado do Rio de Janeiro; 2012.

25.Berardinelli LMM, Santos I, Santos MLCS, Lima TCL, Missio AC, Berardinelli LM. Identificando vulnerabilidade para complicações cardiovasculares em idosos: uma estratégia para o cuidado. Rev enferm UERJ. 2011; 19:541-6.

26.Montez JK, Friedman EM. Educational attainment and adult health: under what conditions is the association causal? Soc Sci Med. 2015; 127:1-7.

27.Felipe GF, Silveira LC, Moreira TMM, Freitas MC. Presença implicada e em reserva do enfermeiro na educação em saúde à pessoa com hipertensão. Rev enferm UERJ. 2012; 20:45-9.

28. Landim CAP, Milomens KMP, Diógenes MAR. Déficits de autocuidado em clientes com diabetes mellitus gestacional: uma contribuição para a enfermagem. Rev Gaúcha Enferm. 2008; 29:374-81.

29. Luna NSA, Baeza MR, Castell EC, Santos FC, David HL, Castillo MMA. Intervención educativa: implementación de la agencia de autocuidado y adherencia terapéutica desde la perspectiva del paciente diabético. Rev enferm UERJ. 2013; 21:289-94.

30. Manoel MF, Marcon SS, Baldissera VDA. Estratégias educativas para pessoas com hipertensão arterial e Diabetes Mellitus. Rev enferm UERJ. 2013; 21:403-8.

31.Xavier ATF, Bittar DB, Ataíde MBC. Crenças no autocuidado em diabetes: implicações para a prática. Texto contexto - enferm. 2009; 18(1):124-30. 